\title{
Simulation Analysis of Optimal Excitation Control for Mobile Power Station
}

\author{
Yu-tong Wang ${ }^{1,}$, , Tian-zhang Shao ${ }^{1}$, Zhi-feng $\mathrm{Gu}^{1}$,Hui-tong $\mathrm{Gu}^{2}$ \\ ${ }^{1}$ Ordnance Engineering College, Shijiazhuang 050003, China; \\ 2 Jun Feng Kechuang Technology Co.Ltd, Shijiazhuang 050065, China. \\ a1003709505@qq.com
}

Keywords: mobile power station, synchronous generator, optimal excitation control.

\begin{abstract}
Through the modeling and simulating of the synchronous generator, the mechanical and electrical properties of the input load are analyzed. Based on the linear control theory, the optimal excitation control of a mobile station is studied. By comparing the optimal excitation control with the traditional control results, it shows the superiority of the optimal excitation control of the synchronous machine.
\end{abstract}

\section{Introduction}

In this paper, the optimal excitation control is adopted for the model of the power system of the mobile station. The optimal excitation control is multiple output bias generator according to the optimal feedback coefficient and the formation of superimposed control variables, state variables and feedback variables are selected as $\Delta V_{t}$, speed deviation $\Delta \omega$ and angle deviation $\Delta \delta$, the feedback gain coefficient can be objective function by linear state equation of a generator operation point and two quadratic performance index minimum solution get, so that the state control reached in the design of optimal operating point.

\section{Analysis of Optimal Excitation Control Method}

Full state optimal excitation control, which is based on the original single control $V_{t}$, increases the control variables of the $\Delta \omega$ and $\Delta \delta$. Dynamic linearization equation $\left(\omega_{0}=1\right)$ for power system of power station:

$$
\left[\begin{array}{c}
\Delta \dot{\delta} \\
\Delta \dot{\omega} \\
\Delta \dot{V}_{t}
\end{array}\right]=\left[\begin{array}{ccc}
0 & 1 & 0 \\
-\frac{\omega_{0}}{H} S_{E^{\prime}} & -\frac{D}{H} & -\frac{\omega_{0}}{H} R_{E^{\prime}} \\
\frac{K_{6}\left(S_{E}-S_{E^{\prime}}\right)+K_{5} T_{d 0} R_{E}}{T_{d 0} R_{E}} & K_{5} & -\frac{1}{T_{d}^{\prime}}
\end{array}\right]\left[\begin{array}{c}
\Delta \delta \\
\Delta \omega \\
\Delta V_{t}
\end{array}\right]+\left[\begin{array}{c}
0 \\
0 \\
\frac{K_{6}}{T_{d 0}}
\end{array}\right] \Delta E_{f}
$$

The type corresponding to the state equation : $\dot{X}=A X+B U$, Among them: $X=\left[\Delta \delta, \Delta \omega, \Delta V_{t}\right]^{T}, U=\Delta E_{f}, B=\left[\begin{array}{lll}0 & , 0 & , K_{6} / T_{d 0}\end{array}\right]^{T}$. In the optimal excitation control system, the performance of the quadratic is specified: $J=\frac{1}{2} \int_{0}^{\infty}\left(X^{T} Q X+U^{T} R U\right) d t=J_{\min }$. According to the principle of optimization, the optimal feedback gain matrix is obtained: $A^{T} P+P A-P B R^{-1} B^{T} P+Q=0$. Optimal control can be obtained: $U=\Delta E_{f}=-K X=-K_{\delta} \Delta \delta-K_{\omega} \Delta \omega-K_{v} \Delta V_{t}$. So, The dynamic linearization diagram of the power system becomes as shown in figure 1: 


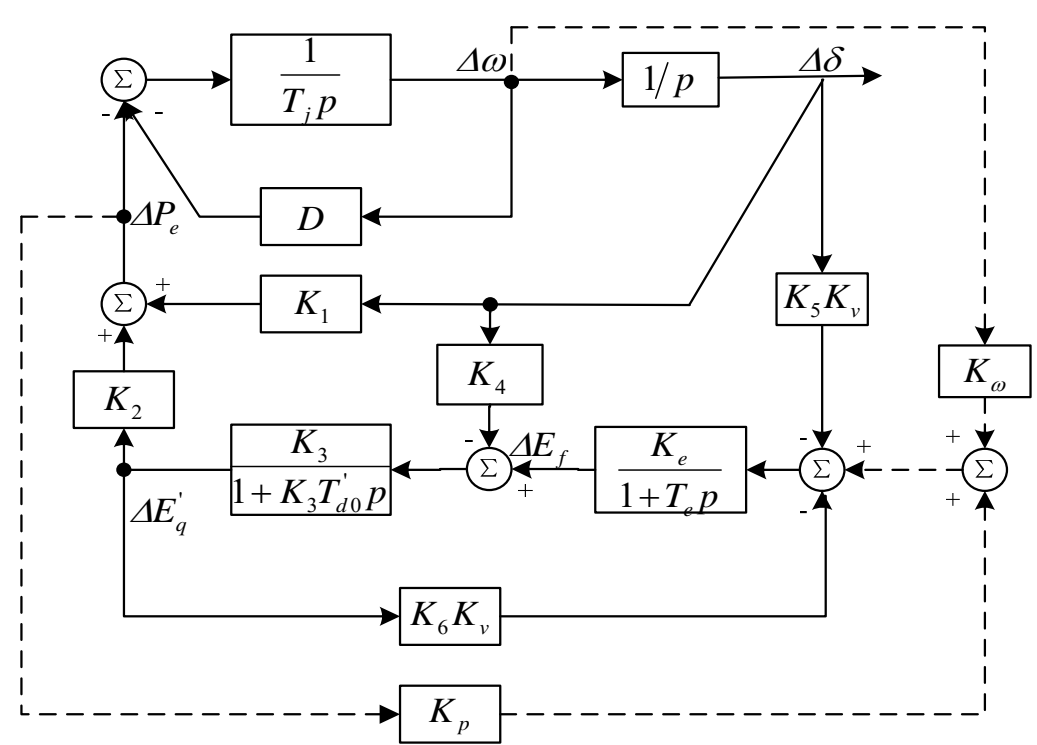

Fig. 1 Optimal Control of power system dynamic linearization block diagram

As can be seen from Figure 1, this system increases the additional two excitation control signals, that is $K_{\omega}$ and $K_{p}$, at the same time, $K_{5}$ and $K_{6}$ all multiply $K_{v}$, the additional torque is:

$$
\Delta T_{e}^{*}=-\frac{K_{2} K_{e}}{\left(1+T_{E} p\right)\left(K_{3}+T_{d 0}^{\prime} p\right)+K_{6} K_{e}} \times G_{o p t}(p) \Delta \omega \approx D_{e}^{*} \Delta \omega+K_{e}^{*} \Delta \delta
$$

Among them: $G_{\text {opt }}(p)=K_{\omega}+K_{p} / T_{j} p$.

It can be seen from the above formula that the excitation control method is used to increase the total damping of the whole system, and the oscillation of the system is suppressed. The way through the performance index function in time domain and automatic compromise the conflicts between the goal of design, dynamic quality and good stability to unity; according to the actual requirements of the object, using the analytical method to obtain the optimal control law, to ensure optimum performance; apply to one or more variables, constant or time-varying linear continuous or discrete systems of many types; good dynamic performance, improve the system robust, adaptability and damping characteristics, improve the static stability limit system. But this system is only applicable to the ideal state with several reasons, the corresponding Riccati has a true solution; local linear method operation point does not take into account the parameters of the running mode is changed, the control performance will be worse, its robust and stability also will be worse.

\section{Comparison of Traditional PID Control And Optimal Control Simulation}

According to the space state equation of the excitation system of the synchronous generator, the model is established in Simulink as shown in Figure 2.

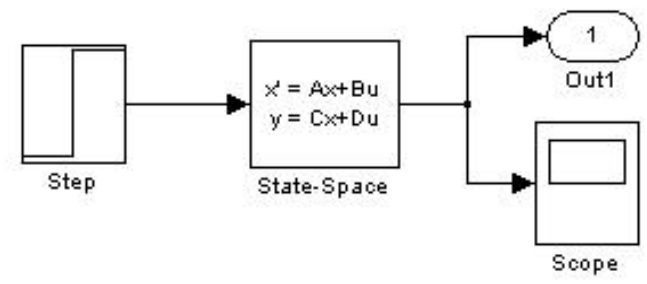

Fig. 2 structure of space state equation of excitation system

The control response of the traditional PID and the optimal excitation are explained separately according to the simulation results.

Parameter of synchronous generator for mobile power station: $x_{\mathrm{s}}=0.74 p . u$. $x_{d}=1.25 p . u . \quad x_{d}^{\prime}=0.221$ p.u. $, x_{d}^{\prime \prime}=0.225 p . u . \quad x_{q}=0.353 p . u . \quad x_{q}^{\prime \prime}=0.152 p . u . T_{d 0}^{\prime}=1.05 s, T_{d 0}^{\prime \prime}=0.05 s$, 


$$
\begin{aligned}
& T_{q}^{\prime \prime}=0.07 \mathrm{~s} . \quad \text { The parameters of the matching voltage regulator are: } T_{r}=32 \mathrm{~ms} \text {, } \\
& T_{a}=6 \mathrm{~ms}, K_{a}=300 .
\end{aligned}
$$

\section{Analysis of Traditional PID Control Step Response}

The transfer function model and the control principle of PID controller, The PID controller is mainly composed of the proportional integral, integral and differential; Combined with the specific application examples, In this paper, we will discuss and analyze the effects of proportional integral controller and proportional derivative controller on the performance of control system:

(1)PI controller: PID control model in the ratio of the constant value of the coefficient, the differential coefficient is zero, that is proportional integral controller; the following system proportional integral control step response curve shown in figure 3.

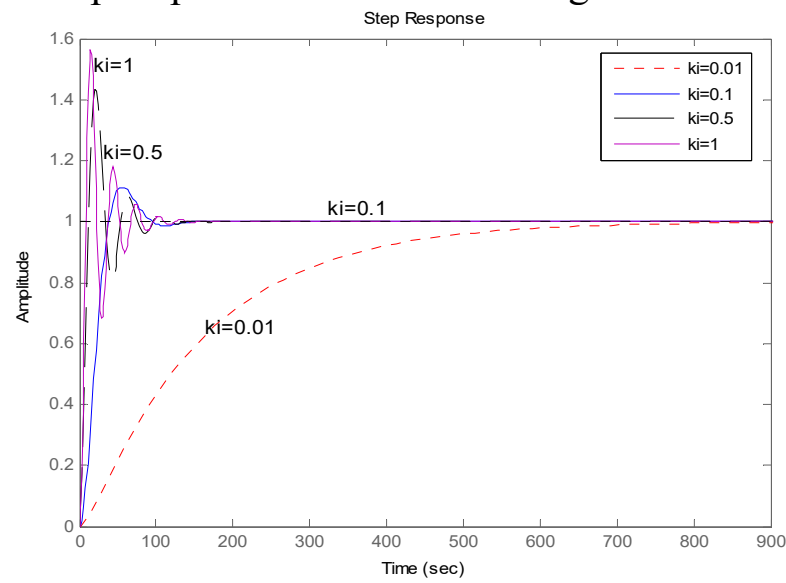

Fig. 3 step response curve of proportional integral controller

With the increase of the integral coefficient proportional integral controller, the control system response speed, adjust the frequency increasing, the maximum overshoot is increased, the system stability is poor, but its steady-state error can be eliminated, which belongs to the non differential regulation.

(2)PD controller: PID control of constant proportional coefficient in the model, the integral coefficient is zero, which is proportional and differential controller; the system of proportional differential control process step response curve as shown in figure 4.

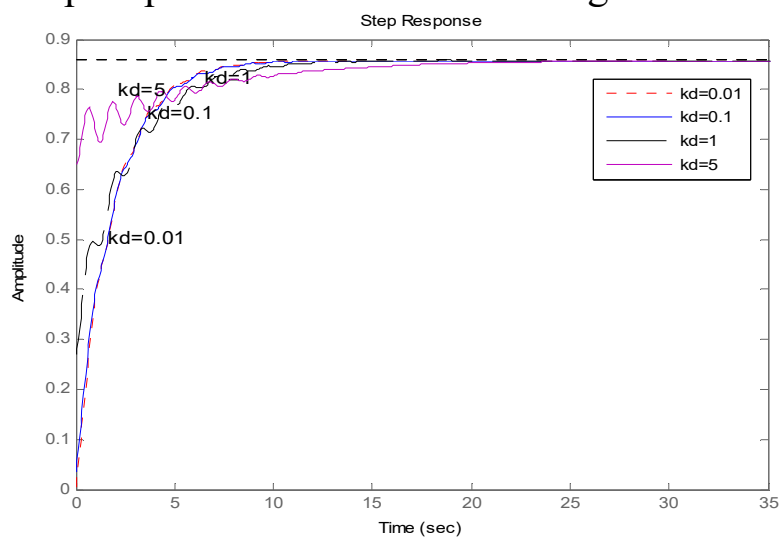

Fig. 4 step response curve of proportional derivative controller

From the results, it can be seen that with the increase of differential coefficient, the control system increases, the maximum overshoot decreases, the system stability is improved, but the steady state error can not be eliminated. 


\section{Optimal Control Step Response Analysis}

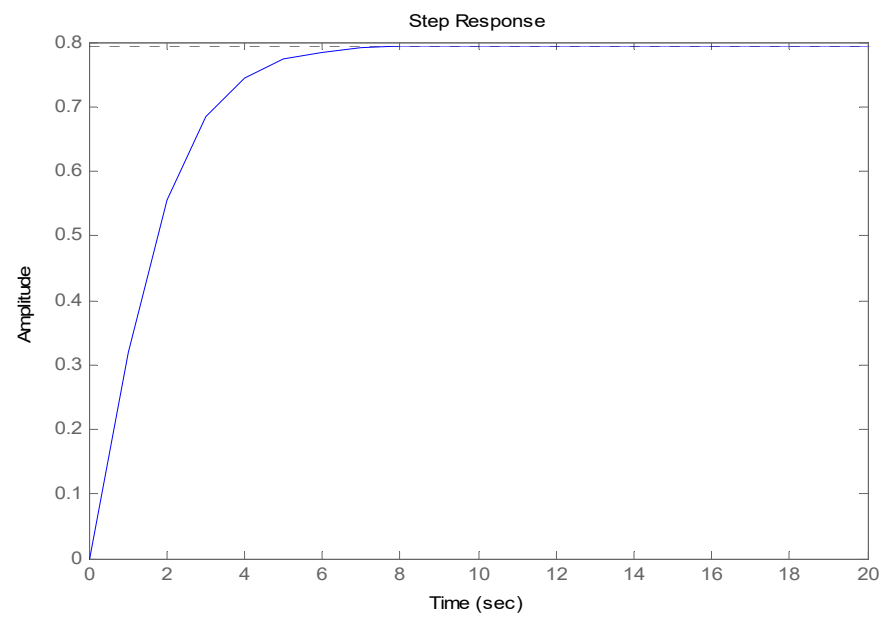

Fig. 5 optimal control step response curve

After optimal state feedback, the system step response curve is ideal. Through the above comparison, the optimal excitation controller can meet the requirements of excitation system optimal control law and control strategy or seek performance index under the condition of the excitation system to deviate from the optimal operating point, the control variable of the system the state variables of the optimal linear combination, this combination can guarantee the square error of the system during the transition of the state the steady-state value of the variable integral minimum system makes the difference is minimal between dynamic response and dynamic response in the optimal operating conditions deviate from the optimal operation conditions under, can effectively adapt to changes in the running state of operation mode in a wide range, can effectively control the system has high dynamic stability limit that is a comprehensive type of excitation control method.

\section{Result Analysis}

In this paper, the optimal operating point is designed as $\delta=10.5^{\circ}, P_{e 0}=0.75$, and the parameters of the synchronous generator are calculated, and the numerical value of each element of $A$ and $B$ matrix is calculated:

$$
\begin{aligned}
A & =\left[\begin{array}{ccc}
0 & 1.0000 & 0 \\
-1.1230 & -0.6250 & -0.2589 \\
-0.9687 & 0.1018 & -0.9901
\end{array}\right] \quad B=\left[\begin{array}{lll}
0 & 0 & 0.8397
\end{array}\right]^{T} \quad Q=\operatorname{diag}[1,100,1000] \\
P & =\left[\begin{array}{ccc}
92.0270 & 1.0362 & -1.3038 \\
1.0362 & 81.2811 & -0.6856 \\
-1.3038 & -0.6856 & 36.2894
\end{array}\right] \quad K=B^{T} P=\left[\begin{array}{lll}
-1.0948 & -0.5756 & 30.4712
\end{array}\right]
\end{aligned}
$$

The expression of optimal control is brought into:

$U=1.0948 \Delta \delta+0.5756 \Delta \omega-30.4712 \Delta V_{t}$

Then the simulation model of the excitation system of the power station is changed to figure 6 . 


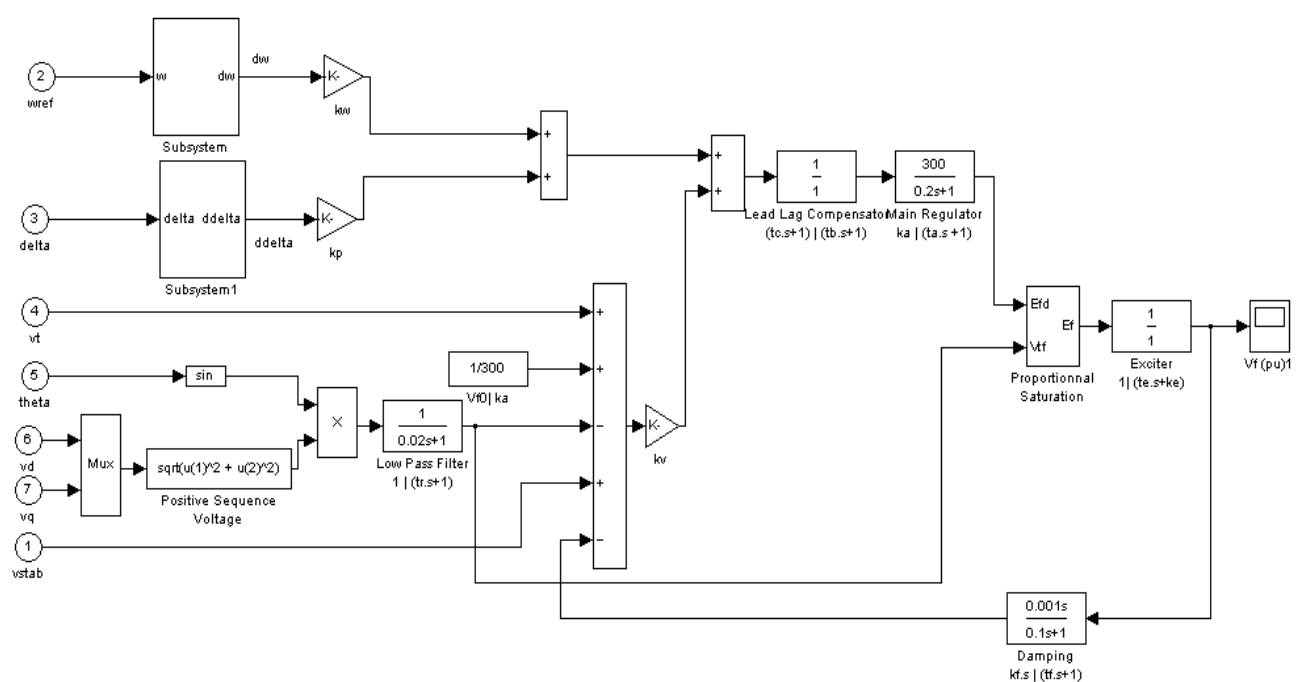

Fig. 6 simulation model of optimal excitation control for mobile power station
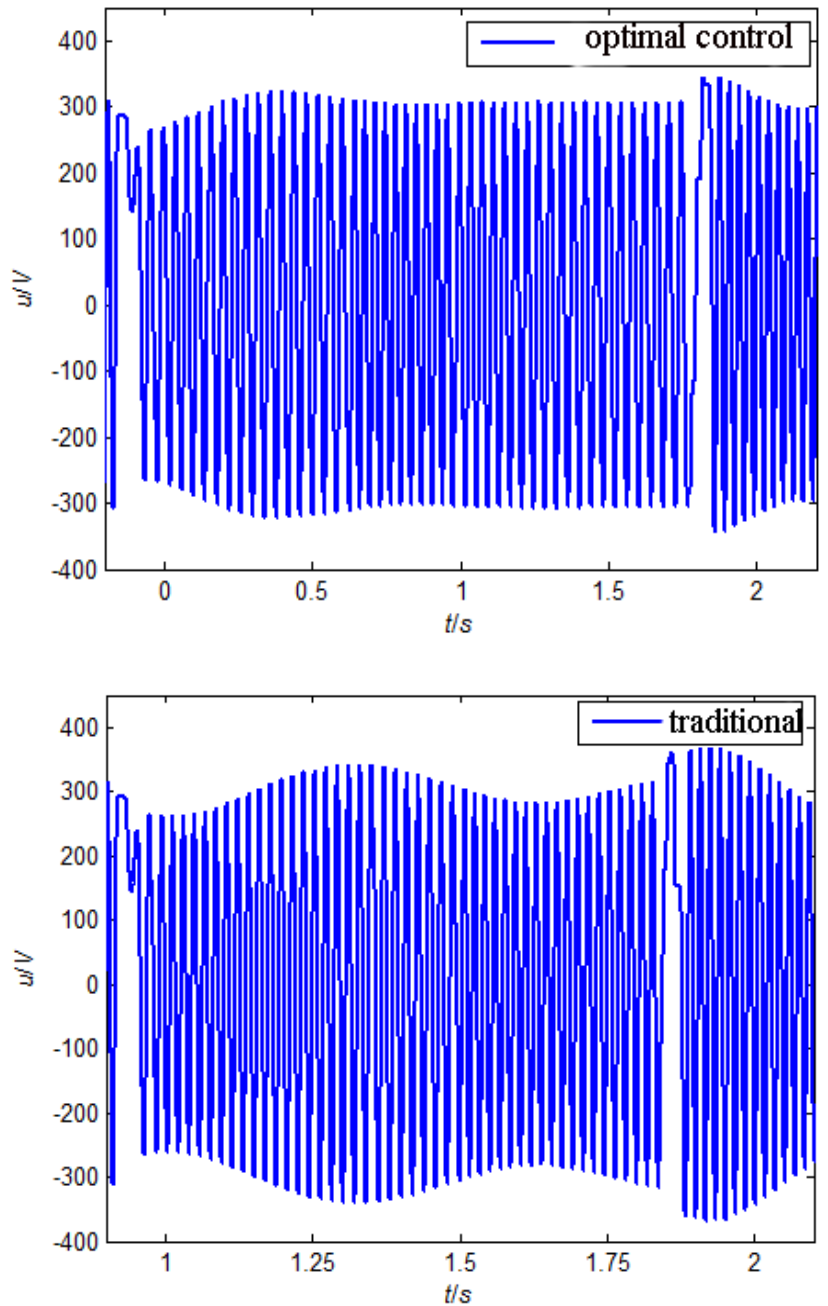

Fig. 7 The optimal control and traditional control terminal voltage comparison

The simulation is $75 \%$ when 1 s load reduction, load recovery in $2 \mathrm{~s}$, Figure 7 shows the comparison of optimal excitation voltage and the traditional control, the test results show that the method for comparing the output voltage of the proposed, has better suppression in optical parametric oscillation and adjust the time effect, the reaction speed, stability for a short time. 


\section{Summary}

Simulation of the traditional power station has carried on the linear dynamic system, and from the state of linearization, get system on the basis of the load is constant and the excitation time constant, the total damping of the system is conserved, analyzes its characteristic root, get rapidly under the condition of excitation system in the high magnification appears low frequency oscillation. This paper for mobile power station adopts the whole state of power system optimal excitation control, make dynamic quality and stability between had good unity.

\section{References}

[1] CHENG Qi-ming, HU Xiao-qing, ZHOU Hui-yun, et al. Optimal control simulation of synchronous genertor excitation system[J]. Journal of Shanghai University of Electric Power, 2011,27(3):275-279.

[2] LING Yan, Design and simulation of excitation system based on linear optimal control theory[J]. The World of Inverters, 2007(7):43-46.

[3] HE Wei-chao, YAO Qiang, Research and simulation of synchronous motor's optimal excitation control system[J].Jiangsu Electrical Apparatus,2011(7):9-13.

[4] YUAN Qi-wei, DU Hong-ji, Design of optimal excitation control system based on Matlab[J]. Industrial Control Computer, 2011,24(9):56-57.

[5] TAO Yu-bo, YANG Cheng-zhi,Research on optimal controller of generator excitation system[J]. Science Technology and Engineering,2011,11(1):35-38,45.

[6] Morioca Y, Tomiyama K, Arima H, etal. System separation equipment to minimize power system instability using genera-tor's angular-velocity measurements[J] . IEEE Trans PowerDelivery, 1993, 8 (3): 941-94

[7] LU Qiang, WANG Zhonghong, HAN Yingduo. Optimal Control of Transmission Power System [M]. Beijing:Science Press, 1982. (in Chinese)

[8] CHANG Xianrong, ZHANG Xiaobin, PAN Yunjiang, et al.Design of integral linear optimal excitation control [J].Automation of Electric Power Systems, 1996, 20(2): 13-17. (in Chinese) 